\title{
Description du mâle de Mexistenasellus parzefalli (Crustacea Isopoda Asellota cavernicole du Mexique) et observations sur cette espèce
}

\author{
Guy MAGNIEZ* \\ GÉNÉRALITÉS
}

Dans cette même revue, j'ai eu l'occasion de décrire, en 1972, les femelles de deux espèces nouvelles de Stenasellidae (Mexistenasellus parzefalli et $M$. wilkensi). Ces Crustacés avaient été capturés dans les collections d'eau de la Cueva del Huizache (située près de Micos, à $27 \mathrm{~km}$ au N-W de Ciudad-Valles, état de San-Luis Potosi, Mexique), par les Locteurs Parzefall et Wilkens, de l'Université de Hambourg. Le lot ne comprenait que $2 q$ de $M$. parzefalli et $1 q$ de $M$. wilkensi, mais il était nécessaire de signaler l'existence de ces Sténasellides, cette famille d'Asellotes souterrains n'étant connue dans le Nouveau-Monde que depuis fort peu de temps (Cole et Minckley 1972). Au début de 1973, le Dr. Horst Wilkens, que je remercie très vivement de sa confiance, m'a fait parvenir un lot plus important d'Isopodes aquatiques de même provenance. La composition de ce lot est la suivante:

1. 8 grands Cirolanides apigmentés et anophtalmes, appartenant au genre Speocirolana Bolivar. Il s'agit vraisemblablement de Sp. bolivari (Rioja, 1951). D'après Reddell et Mitchell (1971), les deux espèces du genre: Sp. pelaezi (Bolivar, 1950) et Sp. bolivari (Rioja, 1951), sont présentes dans toute une série de grottes des états de San-Luis Potosi et de Tamaulipas (Mexique central). Il est intéressant de noter, à la Cueva del Huizache, l'association de trois grandes espèces d'Isopodes cavernicoles aquatiques.

2. 19 adulte de $15,5 \mathrm{~mm}$ de Mexistenasellus wilkensi. Il importera d'effectuer de nouvelles prospections dans les eaux de la grotte, pour découvrir le mâle de cette magnifique espèce.

3. 26 Mexistenasellus parzefalli adultes et subadultes, soient:

- 5 o de 13,$2 ; 12,5 ; 11,2 ; 7,7$ et $7,5 \mathrm{~mm}$,

-3 immatures de 8,$8 ; 8,0$ et $7,7 \mathrm{~mm}$,

- 14 9 adultes au repos génital (oostégites rudimentaires), de 14,0;

13,$9 ; 13,1 ; 13,0 ; 12,5 ; 12,4 ; 11,2 ; 11,1 ; 11,0 ; 9,9 ; 9,4 ; 9,2 ; 7,9$ et

$7,7 \mathrm{~mm}$,

-4 9 à marsupium vide (oostégites fonctionnels), de 14,0;13,0; 13,0 et $12,5 \mathrm{~mm}$.

*Laboratoire de Biologie Animale et Générale, Faculté des Sciences, 6, Bd. Gabriel, 21 DIJON, France. 
Il est donc désormais possible:

a. De décrire le ơ de l'espèce et en particulier d'étudier l'aspect des pléopodes I et II ¿, dont l'intérêt, chez les Asellotes, est considérable (à ce jour, sur les 4 espèces de Stenasellidae du Nouveau-Monde, seul le ơ de la forme-type Mexistenasellus coahuila Cole et Minckley avait été capturé),

b. De compléter la description de l'espèce, les fouets de l'antennule et de l'antenne du type étant absents,

c. D'observer les caractères sexuels temporaires de la $q$ en intermue de reproduction (modifications du maxillipède en particulier).

\section{DESCRIPTION DU ơ DE MEXISTENASELLUS PARZEFALLI}

1. Corps: Tête, péréion et pléon ont très sensiblement le même aspect chez la $q$ et aucun dimorphisme sexuel notable ne les atteint. Nous retrouvons, en particulier, le grand développement des régions pleurales des péréionites, qui va de pair avec une réduction et l'ankylose des coxopodites des péréiopodes (Magniez 1972, p. 21, fig. $1 \mathrm{~J})$. La même particularité existe, je le rappelle, chez des espèces de l'Ancien Monde, comme Stenasellus gjorgjevici de Serbie, Johannella purpurea d'Algérie ou Parastenasellus chappuisi d'Afrique occidentale. Ce n'est donc pas un caractère à valeur générique, puisque les autres Mexistenasellus ( $M$. coahuila et $M$. wilkensi) possèdent au contraire, des péréionites à pleurons étroits, laissant apparaître l'expansion épimérale d'un coxopodite bien développé. La taille du ơ de $M$. parzefalli serait, en moyenne, très légèrement inférieure à celle de la $\uparrow$, ce qui est normal.

2. Appendices céphaliques: Les antennules atteignent $2,7 \mathrm{~mm}$ sur un ơ de $12,5 \mathrm{~mm}$. Le fouet comporte 14 articles, dont les 7 distaux portent chacun une grande lame olfactive hyaline de 160 microns de longueur (fig. 1A). La hampe de l'antenne atteint $3 \mathrm{~mm}$ pour le même individu et son troisième article porte le même

\section{PLANCHE 1:}

$\mathrm{A}-\quad$ Mexistenasellus parzefalli ơ de $12,5 \mathrm{~mm}$ : extrémité d'une antennule; $\mathrm{e}=100$ microns.

$\mathrm{B}-\quad M$. $P$. o de $11,2 \mathrm{~mm}$ : maxillipède gauche, face sternale; $\mathrm{b}=$ basipodite; $\mathrm{c}=$ coxopodite $; \mathrm{e}=400$ microns.

C - $\quad$ M. p. $\quad$ de $13 \mathrm{~mm}$ au repos génital: le coxopodite (c) du maxillipède porte un petit lobe charnu interne (1) et un autre externe (2); e $=400$ microns.

$\mathrm{D}$ - $\quad M . p$. 9 à marsupium de $13 \mathrm{~mm}$ : maxillipède droit, face sternale; les deux lobes (1) et (2) se sont temporairement transformés en deux grandes lamelles ovalaires, à aspect d'oostégites. Seule la pièce interne (1) existe chez Stenasellus virei et les autres formes européennes; $\mathrm{e}=400$ microns. Les soies polymorphes du basipodite et les soies lisses de l'endopodite ont été volontairement omises sur ce schéma, ayant été représentées antérieurement (Magniez 1972).

$\mathrm{E}-\mathrm{F}$ - Maxillipède de la $\uparrow$ ovigère $(\mathrm{E})$ et du $\sigma^{\circ}(\mathrm{F})$ de Caecosphaeroma burgundum Dollfus, d'après Husson et Daum (1954), montrant la similitude des caractères sexuels temporaires + du maxillipède chez le Sténasellide mexicain et le Sphéromien cavernicole européen.

G - Une des soies sensorielles ramifiées de la marge tergale du basipodite des péréiopodes II-VII de Mexistenasellus parzefalli $; \mathrm{e}=200$ microns. 


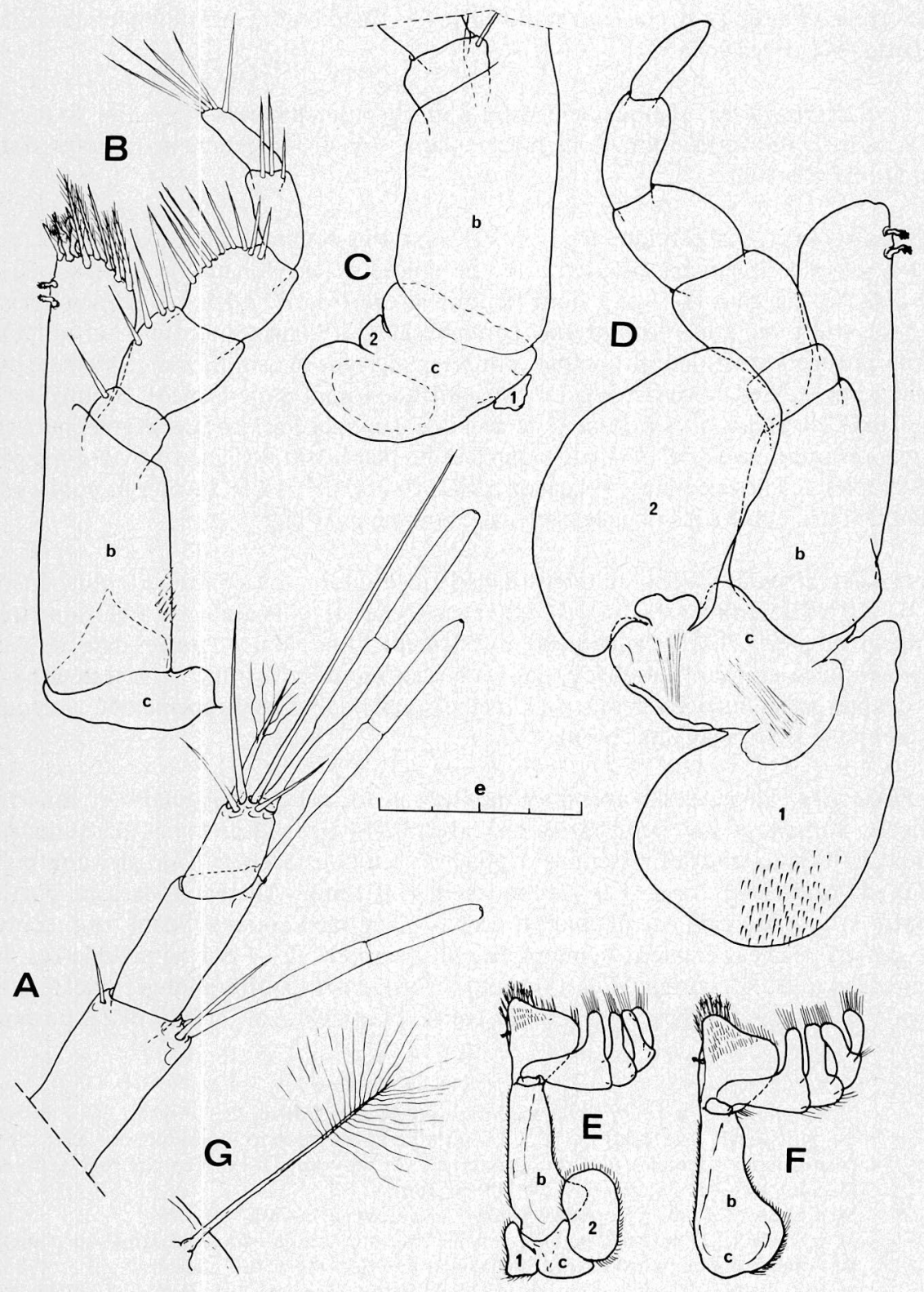


minuscule exopodite unisétigère que la 9 . Le fouet de l'antenne atteint $8 \mathrm{~mm}$. Il est très grêle et fragile. Il compte 80-90 articles. Mandibules, maxillules et maxilles identiques à celles de la $q$.

3. Le maxillipède est typiquement celui d'un Sténasellide, sans épipodite. Celui de la $q$, subissant des transformations spectaculaires lors de l'intermue de reproduction, est étudié plus loin.

4. Péréiopodes: Les péréiopodes II à VII sont très sembables à ceux de la 9 . La marge tergale de leur basipodite porte une rangée de très longues soies sensorielles de 200-300 microns (fig. $1 \mathrm{G}$ ), dont le nombre croît de $\mathrm{P} 2$ à $\mathrm{P} 4$. Les péréiopodes I restent courts et non locomoteurs, comme chez la $q$, mais montrent un notable dimorphisme sexuel: leur propodite est nettement plus massif. Sa marge sternale est mieux armée. Les 5 tiges ensiformes proximales ne sont pas crénelées, comme pour la $\$$; mais elles sont suivies d'une série de 11-12 lames acérées contiguës, inclinées en direction proximale (fig. $2 \mathrm{H}$ ), alors que leur emplacement est, chez la $\$$, occupé par de simples écailles pectinées (Magniez 1972, fig. 1G). L'ongle du dactylopodite est doublé d'une épine sous-unguéale sternale, comme pour la $q$.

5. Papilles génitales: Elles s'insèrent dans l'angle interne du coxopodite du péréiopode VII correspondant, comme c'est la règle chez les Sténasellides. Elles sont très longues et grêles. Elles s'appliquent distalement l'une contre l'autre, dans le plan médian, juste en avant des pléopodes I. Leur extrémité se termine en biseau et peut se coapter avec l'orifice afférent de l'organe copulateur situé du même côté, lorsque le pléopode II se rabat vers l'avant.

6. Pléopodes: Pléopodes I avec protopodite massif, subquadrangulaire et sans rétinacle. Son exopodite étroit et allongé, avec 12-14 tiges lisses sur la marge distale (fig. 2J). Ces appendices ressemblent plutôt à ceux des Stenasellidae africains qu'à ceux de Stenasellus virei. Les pléopodes II sont longs. Au repos, ils sont portés contre la face sternale des opercules, mais le pléopode I correspondant, trop court, ne protège pas entièrement l'endopodite du pléopode II, ce qui rappelle le cas du genre congolais Metastenasellus (Magniez 1966). Protopodite allongé, une fois et demie plus long que large. Exopodite court, biarticulé, son article distal portant

\footnotetext{
PLANCHE 2:

H - Mexistenasellus parzefalli ơ de 12,5 mm: péréiopode I droit, face tergale. Le propodite est plus renflé que celui de la + . La série de lames quasi-jointives qui suit les 5 tiges ensiformes proximales de la marge sternale du propodite n'existent pas chez la $\subsetneq$ (cf. Magniez 1972, p. 21, fig. 1G); e $=200$ microns.

$\mathrm{J}-\quad M . p .0 \mathrm{de} 13,2 \mathrm{~mm}$ : pléopode I gauche, face sternale; $\mathrm{e}=400$ microns.

$\mathrm{K}-\quad M$. p. ô de 13,2 mm: pléopode II gauche, face sternale. L'orifice proximal ou afférent de l'organe copulateur (O.P.) est signalé par une flèche; $\mathrm{e}=400$ microns.

L - Moitié distale du second article de l'endopodite (organe copulateur) du pléopode précédent. Les longues soies (300 microns environ), s'insèrent sur un mamelon interne de la paroi du canal. Elles existent, quoique moins développées, chez d'autres Sténasellides, comme Stenasellus breuili Racovitza; e $=200$ microns.
} 


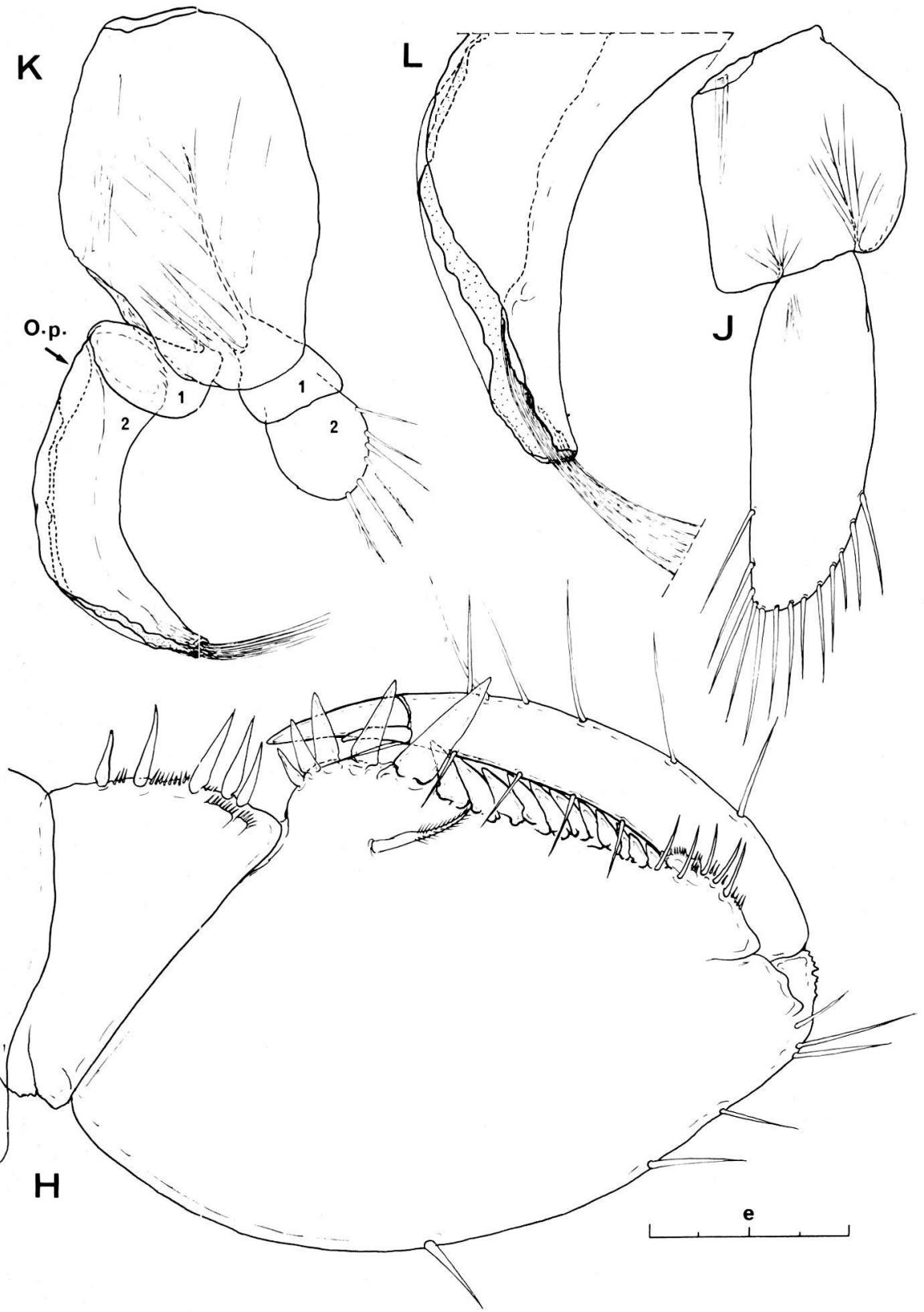


quelques soies, les proximales lisses, les distales plumeuses (fig. 2K). Endopodite biarticulé (ce qui distingue les Stenasellidae des Asellidae). Son article proximal court. Article distal (organe copulateur) très long et falciforme. Il semble constitué d'une pièce foliacée (en forme de feuille de Saule, par exemple), qui se serait repliée longitudinalement autour de sa nervure. Les deux bords viennent s'affronter du côté interne, mais sans se souder. Dans la partie proximale, ils restent écartés, ménageant un infundibulum où la papille génitale peut s'engager (fig. $2 \mathrm{~K} ; \mathrm{O}$. P. = orifice proximal ou afférent). Dans la partie distale, ils ménagent un orifice terminal ou efférent. L'article forme ainsi un conduit imparfait, ouvert à ses deux extrémités et restant fendu selon sa génératrice interne. Dans la portion distale de ce canal existe une protubérance interne, sur laquelle sont insérées de très longues soies ( 15 à 20 au plus), légèrement ramifiées distalement. Elles peuvent atteindre 300 microns de long et forment un long pinceau qui accroît considérablement la longueur de l'organe copulateur (fig. 2L). Un tel dispositif, dont le rôle doit être de faciliter la conduction des spermatozoïdes vers les voies génitales de la \&, existe chez d'autres Sténasellides, (Stenasellus breuili Racovitza, 1924, d'Espagne, par exemple), mais nulle part avec un tel développement. A lui seul, ce caractère suffit à caractériser l'espèce. Chez Mexistenasellus coahuila, qui est de taille bien plus réduite, la protubérance interne distale de l'organe copulateur existe également, mais glabre.

Le pléopode II de $M$. parzefalli ơ est donc celui d'un Stenasellidae typique. Son endopodite biarticulé est toutefois caractérisé par une nette réduction en longueur du premier article, compensée par le développement inhabituel du second. Une telle tendance évolutive existe aussi dans les genres africains Metastenasellus et Parastenasellus plutôt que chez Stenasellus. Ce pléopode II ơ est nettement différent de celui de $M$. coahuila, espèce-type du genre américain, bien que l'organe copulateur, largement ouvert latéralement dans les deux cas, soit d'aspect primitif. Il sera intéressant de connaître le pléopode II ơ des deux autres espèces de Mexistenasellus, dont la $q$ est seule connue, pour effectuer des comparaisons plus poussées.

Les pléopodes III, IV et $\mathrm{V}$ sont pratiquement identiques à ceux de la $\%$. Les uropodes possèdent le même aspect exceptionnel, par suite d'un développement en longueur du protopodite aussi important que celui des deux rames, ce qui distingue M. parzefalli de tous les autres Sténasellides connus.

\section{OBSERVATIONS COMPLÉMENTAIRES SUR L'ESPÈCE}

1. Un rapide examen d'un individu détèrioré a montré que l'espèce possède une seule paire de caecums entériques, comme les Sténasellides de l'Ancien Monde. Ceci distinguerait donc les Stenasellidae des Asellidae qui, eux, semblent toujours munis de 2 paires de caecums.

2. Sur la taille de puberté: Les 5 ò du lot ont tous atteint la conformation définitive de leurs pléopodes I et II. Les plus petits $(7,5$ et $7,7 \mathrm{~mm})$, sont donc déjà des adultes. Parmi les $q$, au contraire, 3 , mesurant 7,7 à $8,8 \mathrm{~mm}$, sont encore dépour- 
vues d'oostégites. Il semble que ces organes apparaissent pour une taille comprise entre 8 et $9 \mathrm{~mm}$, chez $M$. parzefalli. Ceci confirme les observations réalisées sur Stenasellus virei et les formes voisines: l'aspect adulte n'est atteint, chez les $\$$, que pour une taille déjà élevée, qui dépasse la moitié de la taille maximale de l'espèce. La période de vie juvénile est donc certainement très longue.

\section{Caractères sexuels temporaires de la femelle:}

Les oostégites fonctionnels, portés par les $q$ adultes, en intermues de repos génital, sont très comparables à ceux des autres Sténasellides, c'est-à-dire relativement longs.

Dans le lot $q$, se trouvent 4 grands individus à marsupium vide, qui avaient vraisemblablement libéré leur portée au moment de la capture. Les oostégites fonctionnels qui forment la poche incubatrice (portés à la base des péréiopodes I-IV) sont semblables à ceux des autres Sténaselles, mais, c'est sur les maxillipèdes qu'une observation intéressante a pu être faite:

\section{Les oostégites du maxillipède de la femelle gestante:}

On sait que le maxillipède des Asellidae est muni d'un grand épipodite subquadrangulaire induré et muni de soies marginales. Cette production coxopodiale est permanente et identique chez les ot et les $\$$. Le maxillipède des Stenasellidae est totalement dépourvu de cette formation et rappelle plutôt celui de certains Cirolanides, des Sphéromiens ou des Microcerberidae. La constance de ce caractère a été utilisée pour la diagnose différentielle des deux familles d'Aselloidea: Asellidae et Stenasellidae.

Chez les Asellides, le maxillipède de la $q$ adulte porte, en plus, un petit lobe coxopodial interne qui, lors de la mue parturielle, se développe en une palette sétigère (Wasserstrudelapparat ou Putzapparat des auteurs allemands), cf. Gravier 1931, p. 345 et Balesdent 1964, p. 52. Chez les Sténasellides, le maxillipède de la $q$ adulte porte également ce petit lobe interne, muni d'un muscle moteur. Mais, lors de la mue parturielle, il se développe en une grande lamelle glabre, de même aspect que les oostégites cles péréiopodes I-IV (Racovitza 1924, p. 84).

Chez la $\subsetneq$ gestante de $M$. parzefalli, il se développe sur le maxillipède, lors de la mue parturielle, non pas une, mais deux lamelles à aspect d'oostégite (fig. 2D). La première (1) interne, occupant la position de l'oostégite maxillipédien de St. virei; la seconde, externe, occupant la position de l'épipodite permanent des Asellides. Chez la $q$ de $M$. purzefalli, au repos génital, ces deux formations sont représentées par deux minuscules lobes, situés de part et d'autre du coxopodite du maxillipède (fig. 1C). Le maxillipède du ô en est totalement privé. De telles doubles formations temporaires sont déjà connues sur le maxillipède des $q$ gestantes de certains Isopodes Cirolanides et surtout de Sphéromiens cavernicoles, tels que Caecosphaeroma burgundum Dollfus. Elles ont été décrites et interprétées par Husson et Daum (1954). Il sera intéressant, lorsque les captures le permettront, de vérifier si ce curieux caractère sexuel temporaire du maxillipède de $M$. parzefalli $\$$ se retrouve dans les autres espèces du genre américain ou chez d'autres formes tropicales de la famille. Ceci permettrait de savoir si sa signification est d'ordre systé- 
matique ou plus simplement physiologique. Dans ce dernier cas, le grand développement des formations temporaires du maxillipède chez la $q$ gestante pourrait être lié à la nécessité d'assurer aux embryons un renouvellement très intense de l'eau du marsupium, les eaux souterraines tropicales se trouvant à une température élevée et étant particulièrement pauvres en oxygène.

\section{RÉSUMÉ}

Description du ơ du Sténasellide cavernicole mexicain: Mexistenasellus parzefalli Magniez, 1972, provenant de la grotte Huizache, état de San-Luis Potosi, Mexique.

Chez les $q$ à poche incubatrice, le coxopodite du maxillipède porte deux expansions provisoires, à aspect d'oostégite, tandis que chez les $\uparrow$ des Sténasellides européens, on ne trouve que la lamelle interne. Ce caractère sexuel temporaire de la + est connu chez d'autres Isopodes, en particulier le cavernicole Caecosphaeroma burgundum Dollfus.

\section{SUMMARY}

Description of the $\delta$ of the mexican cavernicolous Stenasellid Mexistenasellus parzefalli Magniez, 1972, from Huizache Cave, San-Luis Potosi State, Mexico.

In the $q$ with a brood pouch, the coxopodit of the maxilliped contains two provisional, oostegit-like plates, whereas the ovigerous $q$ of european Stenasellids have only the inner one.

This temporary sexual $q$ character is known in other Isopods, such as the cavernicolous Caecosphaeroma burgundum Dollfus.

\section{BIBLIOGRAPHIE}

BALESDENT, M.L. 1964. Recherches sur la sexualité et le déterminisme des caractères sexuels d'Asellus aquaticus Linné (Crustacé Isopode). Th. Fac. Sci. Univ. Nancy, 1-231.

COLE, G.A. \& MINCKLEY, W.L. 1972. Stenasellid Isopod Crustaceans in the Western Hemisphere - A new Genus and Species from Mexico - With a review of other North American freshwater Isopod Genera. Proc. Biol. Soc. Washington, 84, 39, 313-326.

GRAVIER, Ch. 1931. La ponte et l'incubation chez les Crustacés. Ann. Sc. Nat. Zool., 10, 14, 303-418.

HUSSON, R. \& DAUM, J. 1954. Sur le dimorphisme sexuel des maxillipèdes de Caecosphaeroma burgundum et sa signification physiologique. C. R. Acad. Sci. Paris, 238, 2121-2123.

MAGNIEZ, G. 1966. Contribution à la systématique des Stenasellinae d'Afrique (Crustacés Asellotes). Int. J. Speleol., 2, 173-190.

MAGNIEZ, G. 1972. Deux Stenasellidae cavernicoles nouveaux de l'Amérique centrale: Mexistenasellus parzefalli $\mathrm{n}$. sp. et Mexistenasellus wilkensi $\mathrm{n}$. sp. (Crustacea Isopoda Asellota). Int. J. Speleol., 4, 19-31.

RACOVITZA, E.G. 1924. Diagnose des genres Asellus et Stenasellus et description de deux Stenasellus nouveaux. Bull. Soc. Sci. Cluj, 2, 81-92.

REDDELL, J.R. \& MITCHELL, R.W. 1971. Studies on the cavernicole fauna of Mexico. Association for mexican caves studies, Bull. No. 4, Austin, Texas, 1-239. 